\title{
Diffraction Studies of Nanocrystals: Theory and Experiment
}

\author{
B. Palosz ${ }^{a, *}$,E. Grzanka ${ }^{a, b}$, S. Gierlotka ${ }^{a}$, S. Stel'makh ${ }^{a}$, \\ R. Pielaszek ${ }^{a, b}$, U. Bismayer ${ }^{c}$, J. Neuefeind ${ }^{d}$, H.-P. Weber ${ }^{e}$ \\ AND W. PALOSZ $f$ \\ ${ }^{a}$ High Pressure Research Center UNIPRESS \\ Sokolówska 29/37, 01-142 Warsaw, Poland \\ ${ }^{b}$ Institute of Experimental Physics, Warsaw University \\ Hoża 69, 00-681 Warsaw, Poland \\ ${ }^{c}$ Mineral-Petrographisches Institut, Uni Hamburg \\ Grindelalle 48, 20146 Hamburg, Germany \\ ${ }^{d}$ HASYLAB at DESY, Notkestr. 85, 22603 Hamburg, Germany \\ ${ }^{e}$ SNBL at ESRF, BP 220, Av des Martyrs, 38043 Grenoble, France \\ ${ }^{f}$ USRA/NASA-Marshall Space Flight Center, Huntsville, Alabama 35812, USA \\ Based on theoretical calculations of powder diffraction data it is shown \\ that the assumption of the infinite crystal lattice for small particles is not \\ justified, leads to significant changes of the diffraction patterns, and may lead \\ to erroneous interpretation of the experimental results. An alternate evalu- \\ ation of diffraction data of nanoparticles, based on the so-called "apparent \\ lattice parameter", alp, is proposed. \\ PACS numbers: $61.46 .+\mathrm{w}$
}

\section{Introduction}

The crystal structure of materials is determined by the combined effect of the long-range forces irrespective of the size of the object. The actual structure of a particle is, however, dependent on its size. This dependence can be ignored for large particles - crystallites, but it must be taken into account when the characteristic dimension of an assemble of atoms is comparable to the effective

* corresponding author; e-mail: palosz@unipress.waw.pl 
radius of the long-range interactions. This applies in particular to nanocrystals which can be as small as a few nanometers only. Nanocrystals belong to a general class of polycrystalline materials and are distinguished as a separate class due to their small size and related unique properties $[1,2]$. Different properties (and behavior) of the surface and interior atoms can be deduced and characterized based on the concept of atomic clusters. According to this theory (which refers to the many-body potential) the hybrid orbitals present in atomic clusters are able to form periodic hybrid orbitals and to arrange the individual atoms into a three-dimensional lattice. To form such orbitals (in the interior of the clusters) a critical size of the cluster (a minimum number of atoms) is necessary. In a finite object there is always a discontinuity of the atomic structure at the surface (and formation of the surface layer/shell) leading to the presence of a surface energy. The effect of the many-body potential in the object on ordering of the outer atomic layers may be expected to decrease monotonically from the interior towards the surface of the particle [1-3]. It is obvious that the atomic structure of the inner boundary of the surface layer must be the same as that of the grain core. The deviation of the layer structure (forming a "buffer zone" [1-3]) from the matrix should increase towards the most external atomic layer. A simple definition of the "surface layer thickness" of a particle is that it is equal to that of the "buffer zone". However, we are not aware of any experimental methods which could be used to measure the as-defined thickness of the surface layer of nanoparticles. To discern between the surface and interior phases of a grain, their physical and/or structural properties must be sufficiently different to be experimentally measurable. In this work we discuss this problem with regard to the capabilities of the powder diffraction techniques.

The properties of nanocrystalline materials are critically dependent on the atomic structure of the constituent grains. A size-dependent atomic structure of nanoparticles may lead to unique properties (like unusual hardness or superplasticity, different melting temperature or luminescence spectra, etc.) of nanomaterials relative to conventional polycrystals of the same substance [4-8]. The surface of individual crystallites apparently plays an important role in this and other phenomena. In conventional polycrystalline materials with micrometer size grains the surface atoms constitute only a small fraction of the total number of atoms and their effect on the overall properties of the material can be ignored. The situation is different in small, nanosize particles where, due to their size, a considerable fraction of the atoms forms the surface of the grain. As shown in Fig. 1, an abrupt termination of a perfect crystal lattice (Fig. 1a) leaves the outmost atoms without some of their neighbors (Fig. 1b) which leads either to a reconstruction of the surface or to a generation of strains through changes of the length of the interatomic bonds (Fig. 1c). Assuming that the surface shell has the thickness of $1 \mathrm{~nm}$, a $10 \mathrm{~nm}$ diameter grain contains $25 \%$ of its atoms in the surface layer, while a $4 \mathrm{~nm}$ grain has already $60 \%$ of such atoms. This shows that, in very small objects (a few $\mathrm{nm}$ 
in diameter), the number of surface-related atoms can exceed the number of the "bulk" atoms. Whichever process (reconstruction or generation of strains) dominates, it may be expected to lead to some unique properties of the materials. It may also be expected that the structural changes at the surface propagate to the depth of several interatomic distances towards the particle center. Therefore, in a meaningful research on nanocrystals the surface layer (shell) should be treated as a separate structural phase relative to the bulk of the grain (the core).

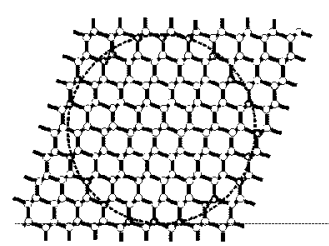

(a)

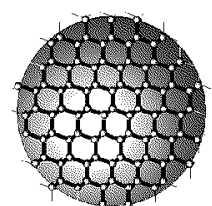

(b)

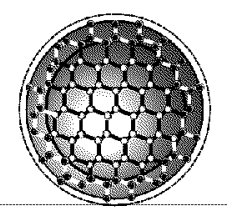

(c)

Fig. 1. Tentative models of the atomic structure of a nanocrystal: (a) single crystal, (b) hypothetical nanocrystal with atoms located at the perfect lattice sites, (c) nanocrystal with a compressed lattice in the surface shell.

Although there is a general understanding in the scientific community that the surface has a significant effect on the physical properties of nanocrystals, the unique properties of these materials are being related to the size of the grains and not to the structure of their surface. A number of literature reports on the dependence of different physical properties on the size of nanoparticles exist [9-15]. There is no doubt that changes of physical properties of materials are related to corresponding changes in their atomic structure, which is clearly demonstrated in phase transition processes. Changes of the atomic structure of a crystal are always reflected by the corresponding changes of its lattice parameters. Therefore, while the specific atomic structures of nanocrystals have not been determined yet, changes of the lattice parameters of nanocrystals (determined from powder diffraction data) relative to those of the bulk crystals have been reported for a variety of nanomaterials, e.g. in metals, $\mathrm{Au}[16,17], \mathrm{Al}[18], \mathrm{Cu}$ [19], semiconductors like CdSe [20], ionic crystals like $\mathrm{NaCl}, \mathrm{KCl}, \mathrm{NaBr}$ and $\mathrm{LiF}$ [21]. Those results were interpreted as "the effect of the grain size on the lattice parameters" and often attributed to the presence of a strong (homogeneous) "internal pressure" caused by the surface stresses (analogous to the surface tension in liquids [22-29]). This "straightforward" interpretation of the experimental findings ignores the fact that the structure of a nanocrystal is not uniform and should be considered either as composed of two distinctive, grain core and shell phases, or as a structure where a large fraction of the atoms at the grain surface is displaced relative to their regular lattice positions (which is equivalent to a presence of strain in the vicinity of the surface). In large (microsized) crystallites the number of atoms located at or in the vicinity of the grain surface is orders of magnitude smaller than the number of 
atoms in the grain core, and their effect on the bulk properties of the materials can be ignored. Also, the contribution of the surface atoms to the diffraction effects measured for such polycrystals in a conventional powder diffraction experiment is negligible. With a decrease in the dimensions of the crystallites down to a few nanometers the number of atoms located at the surface becomes comparable to those in the grain core. Since the surface atoms have a different surrounding and their interatomic distances may differ from those in the bulk material, one set of lattice parameters for a nanocrystal may be inadequate for a unique description of its structure. Conventional diffraction techniques and standard methods of diffraction data analysis are used to characterize the average crystallographic structure of materials through determination of the lattice parameters of the unit cell $[30,31]$. These methods are insufficient to detect and quantify small differences between the actual atomic positions in nanograins and those in a regular (unambiguously defined) crystallographic phase.

In this paper we show that, although conventional diffraction experiments are appropriate for characterization of the crystallographic structure of nanosize crystalline powders, the routine methods of the powder diffraction data elaboration may lead to erroneous interpretation of the experimental results. We show that the description of the crystallographic structure of such materials based on the unit cell is erroneous. Accordingly, application of the lattice parameters concept, when used for characterization of the structure of nanocrystals, has to be modified. We suggest a replacement of the lattice parameters, describing the dimensions of the unit cell, by a set of different values of the "lattice parameters", each associated with (determined from) one individual Bragg reflection, each one at its specific diffraction vector $\boldsymbol{Q}$. We present the preliminary results of the application of our concept of the "apparent lattice parameters" ( $a l p)$ for the elaboration of powder diffraction data of nanocrystals. The concept is demonstrated for nanocrystals with a perfect crystallographic structure, as well as for those with the atomic structure of the surface different than that in the grain core. Some preliminary experimental evidence of the presence of tensile and compressive surface strains in nanocrystalline particles will be shown.

In this work we discuss only the positions of the intensity maxima (i.e. the geometrical aspects of the atomic structure of nanocrystals) through a comparison of the positions of the maxima of the theoretically calculated and experimentally measured powder diffraction patterns. We do not discuss the intensities of the Bragg (Bragg-like) reflections: we assume that all sites are fully occupied (as in a perfect crystal lattice).

\section{Powder diffraction}

Structural analysis of crystalline materials is performed in two basic steps: (i) determination of the geometry of the crystal lattice and (ii) determination of 
the positions of specific atoms in a representative structural unit (the unit cell). Further analysis can determine the deviations of the crystal lattice from the ideal crystal structure $[30,31]$. In this paper we focus our attention on the analysis of the geometry of the lattice of nanoparticles which, as discussed later, requires a more complex description than that sufficient for conventional bulk crystal structures. For a direct comparison of the diffraction patterns obtained using different radiation sources and geometries we present our results on diffraction intensity profiles as a function of the diffraction vector $\boldsymbol{Q}$.

\subsection{Conventional elaboration of powder diffraction data}

The simplest information that can be derived from a diffraction pattern are lattice parameters. They are determined routinely based on the Bragg equation which relates the lattice parameters to the characteristic intensity maxima of the diffraction patterns. A common approach to the elaboration of diffraction data is based on the Bragg approximation (Fig. 2). According to Bragg, if a unit cell represents a structure, the structure can be described as a set of infinite, equally spaced parallel planes (Fig. 2). The incident radiation beam is reflected by the atomic planes. The direction of the scattered beam, $2 \Theta$, is uniquely defined by the relative orientation $(\Theta)$ of the incident beam and the crystal lattice, and the distance $d_{h k l}$ between the identical atomic planes (Fig. 2). There is an unlimited number of families of planes which can be drawn for a given lattice by joining different nodes of the crystal lattice formed by the periodically stacked unit cells. The planes are denoted by the Miller indices $h k l$. Each family of planes gives a characteristic interference maximum: an $h k l$ Bragg reflection located at unambiguously defined position in the $\boldsymbol{Q}$ space (Fig. 2). The locations of the intensity maxima are determined by the specific inter-planar spacings $d_{h k l}$ and the wavelength of the incident beam. Determination of several Bragg reflections (one in the case of a cubic lattice) is sufficient to determine the symmetry, shape, and dimensions of the unit cell [30, 31].
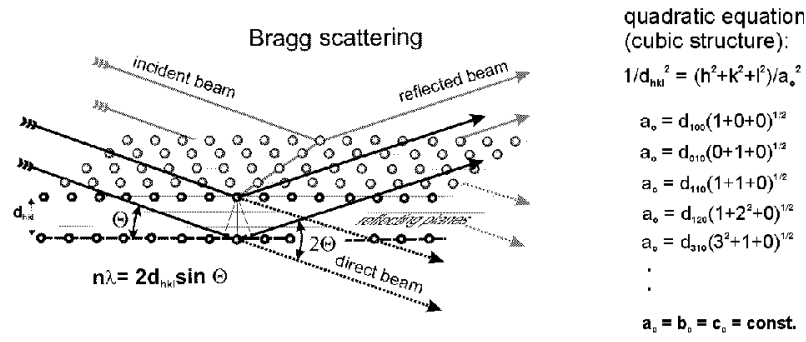

Fig. 2. Diffraction on a perfect crystal lattice: the atoms are located on ideally flat atomic planes.

Routine methods of structural analysis of crystalline materials are based on the simple assumption that a unit cell appropriately represents the material structure $[30,31]$. To characterize accurately such an atomic structure it is sufficient to 
determine (i) the set of the lattice parameters $a, b, c, \alpha, \beta$, and $\gamma$ describing the dimensions and shape of the unit cell and (ii) the location of each of the $i$ atoms in the cell, $x_{i}, y_{i}$, and $z_{i}$. The complete structural analysis (structure refinement) of polycrystalline samples is routinely done with the powder diffraction technique and elaboration of the experimental data with a crystallographic software like those based on the Rietveld methodology [31]. In the Rietveld method it is assumed that the crystal lattice of the material under examination is uniquely defined by the unit cell. An appropriate structural model is assumed and the corresponding diffraction pattern is calculated. The theoretical pattern is compared with the experimental results. In an iterative process the structural model is modified and subsequently refined for the best fit of the calculated and measured diffraction patterns based on the selected fit criteria. Figure 3 shows an example of the "best fit" of the theoretical and measured patterns of a SiC polycrystal; the "perfect" fit would correspond to a straight line of the difference curve.
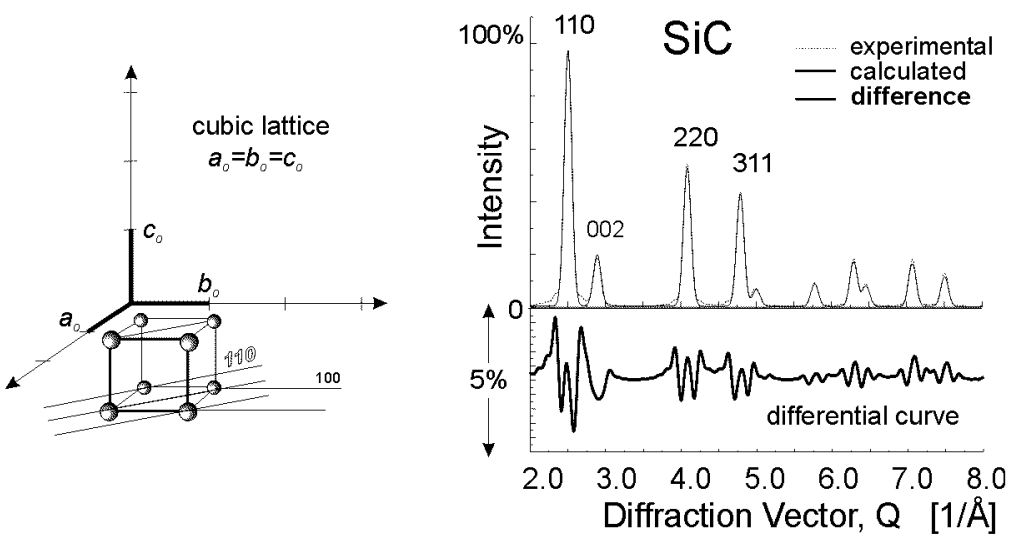

Fig. 3. Theoretical and experimental diffraction patterns of a $\mathrm{SiC}$ polycrystal calculated with the Rietveld refinement program.

The accuracy of determination of the specific values of lattice parameters and atomic coordinates based on a refinement method like the Rietveld program is dependent primarily on the quality of the experimental data. The results of such elaboration are accurate if the fundamental underlying assumptions of a perfect lattice are met, i.e.

(i) the unit cell of the lattice is identical throughout the entire volume of the sample and

(ii) the lattice is an array of points in space in which the environment of each point is identical to each other.

These conditions are never strictly met but, for larger crystallites, the deviations from the ideal model are negligible. When the dimensions of the object are comparable to those of the single unit cell the deviations cannot be ignored. In a 
nanoparticle a large fraction of the atoms is located at the surface and surrounded differently than those in the core (Fig. 1). Thus, a single unit cell cannot represent the entire atomic structure of a nanoparticle. Therefore, the methods of the structural analysis developed for standard crystalline materials may be inadequate for nanosize crystallites.

\subsection{The implications of small crystal size for structural analysis by powder diffraction}

Conventional methods of the analysis of diffraction data were developed with the assumption that the crystal lattices of real objects are infinite in comparison with the dimensions of the representative structural unit chosen for the material. As discussed in Sec. 1 above, this assumption is not valid when the dimensions of the crystal are comparable to the size of the unit cell. For such cases the applicability of conventional methods of the analysis of powder diffraction data (the only diffraction technique that can be used with nanocrystaline materials) needs to be re-evaluated. In our analysis we applied the method of calculation of diffraction patterns introduced in 1915 by Debye [32].

\subsubsection{Theoretical calculations of powder diffraction patterns}

Relatively small number of atoms forming a nanocrystal provides a unique opportunity to design complete, atom-by-atom, models of small crystals. Using such models and the Debye equations, Fig. 4, the corresponding diffraction patterns can be calculated. According to Debye, the intensity of the radiation scattered by an array of atoms is the sum of the beams scattered by every pair of atoms (Fig. 4). The intensities $I(k)$ of radiation scattered by each pair can be integrated over all spatial orientations of a given crystallite yielding the equivalence of the powder diffraction pattern of a large number of randomly oriented grains.

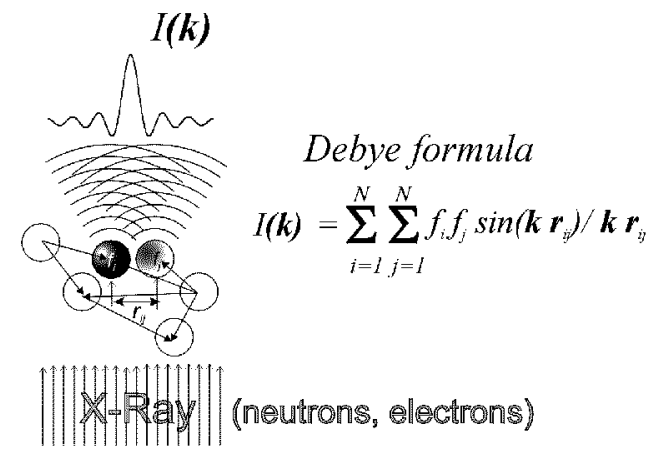

Fig. 4. Scatterring of radiation by an array of atoms; $f_{i}$ and $f_{j}$ are the atomic scattering factors of the $i$-th and $j$-th atom, respectively, $k=4 \pi \sin \Theta / \lambda$ is the scattering vector, and $r_{i j}$ is the distance between the atoms. 
Applying the Debye equation we are able to calculate diffraction patterns for different models of nanocrystalline grains with diameters up to $20 \mathrm{~nm}$ without the assumption of a periodic arrangement of the atoms. An important advantage of a direct calculation of the diffraction patterns is that it allows to implement directly into the model different lattice imperfections like strains, point and planar defects, disorderings, etc. For technical reasons, direct calculations of the diffraction effects can be achieved for relatively small arrays of atoms only. A reasonable CPU time, up to a few hours, is sufficient to complete one calculation of the diffraction pattern for nanoparticles smaller than $20 \mathrm{~nm}$ in diameter [33, 34]. For particles with diameter of $25 \mathrm{~nm}$ and larger the CPU time is of the order of days and weeks.

\subsubsection{Theoretical diffraction patterns of nanocrystals and their elaboration}

To verify the applicability of Bragg approach to nanoparticles, we calculated theoretically (using the Debye functions) the diffraction patterns of crystallographically perfect nanocrystals, and calculated back the lattice constant of the model using the Rietveld refinement program.

The best verification of any method proposed for elaboration of experimental results is an application of the method to a "perfect experimental data". In our case the experiment is simulated by calculating a perfect diffraction pattern using the Debye functions (cf. Sec. 2.2.1).

The elaboration of any diffraction data has to account for two groups of parameters: instrumental and sample-dependent. Our virtual "perfect experiment" does not require any instrument-related corrections. However, in a real laboratory practice the experiments are not perfect and any instrument/geometry imperfection is a source of errors. These instrument imperfections lead to the so-called "zero shift" effect: the real beginning of the diffraction pattern is usually shifted relative to the apparent "zero" position of the experimental setup (Fig. 5a) with the obvious consequences for the accuracy of the experimental results. Standard data elaboration routines evaluate and correct the pattern for the "zero shift" factor.

The second type of experimental errors is due to the properties of the sample itself. The Bragg diffraction relations apply to infinite size crystals and in a model experiment all diffraction peaks have the Gaussian-type shapes. For small crystallites the shape of the peak is no longer Gaussian but a combination of Gaussian and Lorentzian functions (Fig. 5b). Our calculated diffraction patterns were analyzed applying the Rietveld methodology assuming that the reflections have shapes that are a combination of these two basic functions. We used two procedures of the elaboration of our diffraction data:

1. Perfect experiment. In this method, consistent with the "perfect experiment" approach, we did not apply the procedure that corrects for the experimental/instrument imperfections. We refined the as-calculated diffraction data releasing only the lattice parameters and the line shape. The line shapes were fit- 

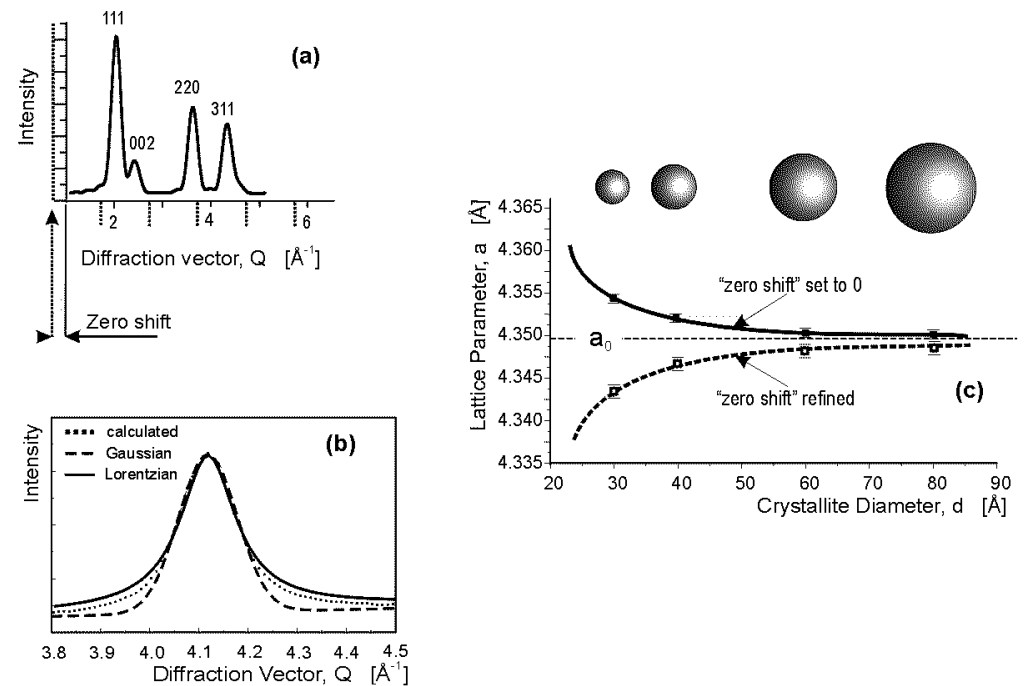

Fig. 5. The results of theoretical calculations of the diffraction effects in nanocrystals of SiC. Lattice parameter of the model, $a_{0}=4.350 \AA$. (a) A typical theoretical diffraction pattern, (b) a representative shape of the diffraction peaks, (c) lattice parameters backcalculated from the theoretical diffraction patterns for different size particles. Full symbols and solid line - the refinement made without correction for the "zero shift"; open symbols and dotted line - the refinement made with correction for the "zero shift".

ted using the pseudo-Voigt function. The calculated values of the lattice parameter for different sizes of the particles are shown in Fig. 5c (full symbols, solid line). Obviously, the procedure yields the lattice parameters different than that of the starting model. In addition, it generated an artificial "dependence of the lattice parameter on the size of the particle".

2. Imperfect experiment. In this case we treated our calculated diffraction data as if they were obtained experimentally and, so, required a correction for the instrument imperfections. Therefore, we refined the lattice parameters releasing the "zero shift" correction in the Rietveld program. The results of this calculation are shown by the open symbols/dashed line in Fig. 5c. As we can see, the "zero shift" correction procedure generated another artefact: despite no real instrumental errors introduced to our model diffraction pattern, the program leads to a change in the value of the calculated lattice parameters relative to the model input value. This effect is apparently even stronger, and opposite in sign than that in the first procedure, so the combined result generates the "dependence of the lattice parameter on the size of the particle" in the opposite direction.

As seen from the above, a mathematically correct, commonly accepted, and widely used Rietveld refinement program yields erroneous results: the lattice parameter backcalculated using this procedure generated an artefact - a "depen- 
dence of the lattice parameter on the grain size". The magnitude of this superficial effect decreases with an increase in the grain size and can be ignored for crystallites $10 \mathrm{~nm}$ in size or larger (Fig. 5c). The reason for the above effects is the non-compatibility of the assumption used in derivation of the Rietveld methodology (infinite atomic planes) and the real nature of the crystals (finite size of real particles). The above limitation of applicability of the Rietveld method has apparently been overlooked by many researchers and seems to have led to incorrect interpretation of the diffraction data of nanomaterials. Our analysis shows that application of routine methods of elaboration of diffraction data requires a very critical assessment of the results. In particular, internal standard should be used to prevent the apparent errors introduced by the "zero shift" correction routine during elaboration of any diffraction results. This is particularly important for crystallites as small as several nanometers.

\section{3. "Apparent lattice parameters" — a novel method of powder diffraction analysis}

A dependence of the results obtained with the Rietveld program on the crystallite size is apparently the effect of the shift of the positions of individual Bragg reflections relative to those corresponding to the perfect lattice. This phenomenon is caused by the approximation of the crystal lattice by a set of infinite atomic planes, which is not justified for nanosize crystals. As a consequence, the Bragg reflections cannot be assigned to a unique value(s) of the lattice parameter(s) so the term "lattice parameter" loses its fundamental meaning. Our analysis of these phenomena discussed in Sec. 2.2.2 led us to a conclusion that the effect of the limited number of atoms in individual nanograins on the diffraction pattern is different for different reflections. As a result, the individual peaks are incoherent to each other ("coherence" means that all Bragg reflections obey the quadratic equation for a single, unique set of the lattice parameters, cf. Fig. 2) and the diffraction pattern evaluation programs that assume coherency lead to erroneous results. Since the lattice parameters of such patterns calculated from different Bragg reflections are not invariant, we developed a new approach to the analysis of the diffraction data based on individual diffraction peaks. For a given Bragg reflection hkl (at the diffraction vector $\boldsymbol{Q}$ ) we calculate the corresponding value of the lattice parameter that we call the "apparent lattice parameter" (alp).

Note: we showed in Sec. 2.2.2 that the quadratic equation, used routinely in every conventional crystallographic program and which serves for refinement of the lattice parameters, is not valid for very small crystallites. Consequently, such an equation cannot be used to calculate the lattice parameters even if the atomic structure of the crystallite has a perfect crystal lattice with three-dimensional periodicity. This means that the methods of diffraction data elaboration based on the Bragg approximation fail: using the Bragg approach and the quadratic 
equation we are unable to obtain the correct information on the atomic structure of a nanocrystal. Under such circumstances the term "Bragg reflection" should then be replaced by the term "Bragg-like reflection".

We propose an application of the Bragg equation and of the quadratic equation for derivation of $d$ values calculated separately for each individual reflection. This way we associate the calculated d-values with the specific values of the diffraction vector $\boldsymbol{Q}$ of a given reflection. From the as-calculated $d$-values we derive the corresponding values of the lattice parameters which we call the "apparent lattice parameters". With this procedure we obtain not the lattice parameters but a set of the alp values and their corresponding diffraction vectors $\boldsymbol{Q}$. (For the sake of simplicity, we illustrate our methodology based on cubic lattices, i.e. the structures defined by only one lattice parameter $\left(a_{0}=b_{0}=c_{0}, \alpha=\beta=\gamma=90^{\circ}\right)$. In a general case, derivation of a set of three independent alp values corresponding to three lattice parameters $a, b$, and $c$ is necessary.) In addition to calculations based on individual Bragg reflections we can also calculate the alp values for a group of several adjacent Bragg reflections in the selected range(s) of the $\boldsymbol{Q}$-values. Such procedure is very useful in the case of a strong overlapping of the reflections, particularly in the high $\boldsymbol{Q}$ range.

The results of the evaluation (using the Rietveld program) of the theoretical diffraction pattern computed for a model of cubic SiC crystal of $8 \mathrm{~nm}$ in diameter for different $\boldsymbol{Q}$-ranges are shown in Fig. 6. The diffraction pattern of the $\mathrm{SiC}$ nanocrystal calculated from the Debye functions and that calculated by the Rietveld program (solid and dashed lines, respectively) are shown in Fig. 6a. The difference between these two patterns is up to $5 \%$ of the maximum intensity of the (111) reflection (Fig. 6b). This is a very large discrepancy, particularly given by the fact that both curves were calculated theoretically for the same, perfect-lattice crystallographic model. The difference between these two calculations is that the Rietveld program assumes that the crystal is infinite and is uniquely represented by the unit cell, while the Debye method accounts only for the existing atoms in the model of the crystallite. The positions of the reflections calculated by the Rietveld program correspond to a perfect crystallographic lattice with one lattice parameter $a$ common for all positions of the Bragg reflections, in accordance with the quadratic equation. The Bragg-like lines calculated from the Debye functions are shifted relative to those corresponding to the Bragg reflections of the "perfect and infinite lattice". This is the main reason of the discrepancy between the patterns shown in Fig. 6b: consistent with the assumption of an unambiguous unit cell, the Rietveld program finds a single lattice parameter which gives the best fit to all Bragg reflections of the pattern. The program has no option to fit individual Bragg reflections. The lattice parameter refined by the Rietveld program is a compromise (the least squares best fit) which is to minimize the discrepancy between the experimentally measured (here: calculated by the Debye functions) and the theoretical pattern of the unit cell used by the Rietveld program. The ad- 
(a)

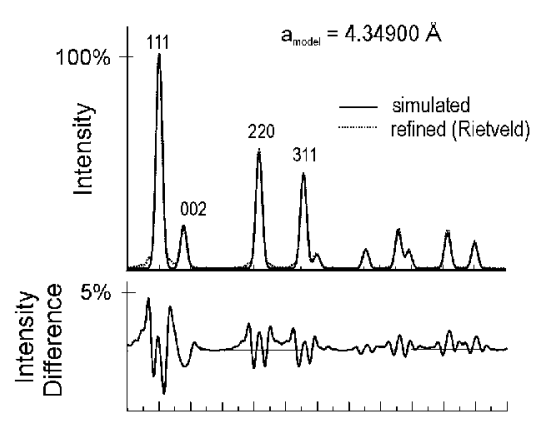

(c)

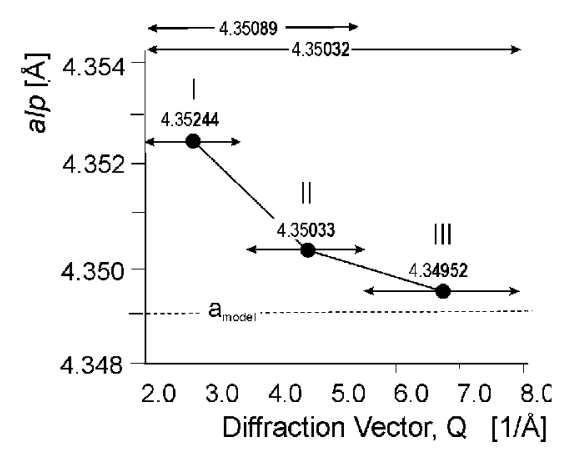

Fig. 6. alp values calculated from the theoretical diffraction pattern of $\mathrm{SiC}$ nanocrystal of $8 \mathrm{~nm}$ in diameter. (a) Solid line - as-calculated diffraction pattern; dotted line - the pattern refined with the Rietveld program, (b) difference in intensity between the as-calculated and refined diffraction patterns, (c) alp values calculated for different ranges of the diffraction vector $\boldsymbol{Q}$.

ditional reason for the discrepancy between the patterns is a complex shape of the Bragg-like reflections of nanocrystals. The Bragg reflections of a uniform-size-grain crystalline powder should have a Gaussian shape. The shift of the Bragg-like reflections of nanocrystals is accompanied by a distortion of the peak symmetry. No simple analytical function describing the shape of the calculated reflections exists. In the Rietveld program we used symmetric line shape functions: Lorentzian, Gaussian and their combination (pseudo-Voigt and Pearson functions).

Figure 6 illustrates the inadequacy of the standard methods for evaluation of diffraction patterns of nanomaterials. The values of the alp parameter calculated for different $\boldsymbol{Q}$-ranges are shown in Fig. 6c. The lattice parameter as-refined for the whole calculated pattern (which is equivalent to the alp parameter computed for the entire diffraction spectra) is $4.35032 \AA$. This value is larger than the lattice parameter of our model, $4.349 \AA, \Delta a / a_{0}=0.03 \%$. The alp value obtained for small $\boldsymbol{Q}$-values (range I) is larger (by $0.08 \%$ ) from that of the model, as are the alp's calculated for greater $\boldsymbol{Q}$ values. The difference between the as-refined alp's and the real $a$ value decreases with an increase in the scattering vector $\boldsymbol{Q}$. The alp values plotted versus the diffraction vector $\boldsymbol{Q}$ follow a monotonic curve which tends to 


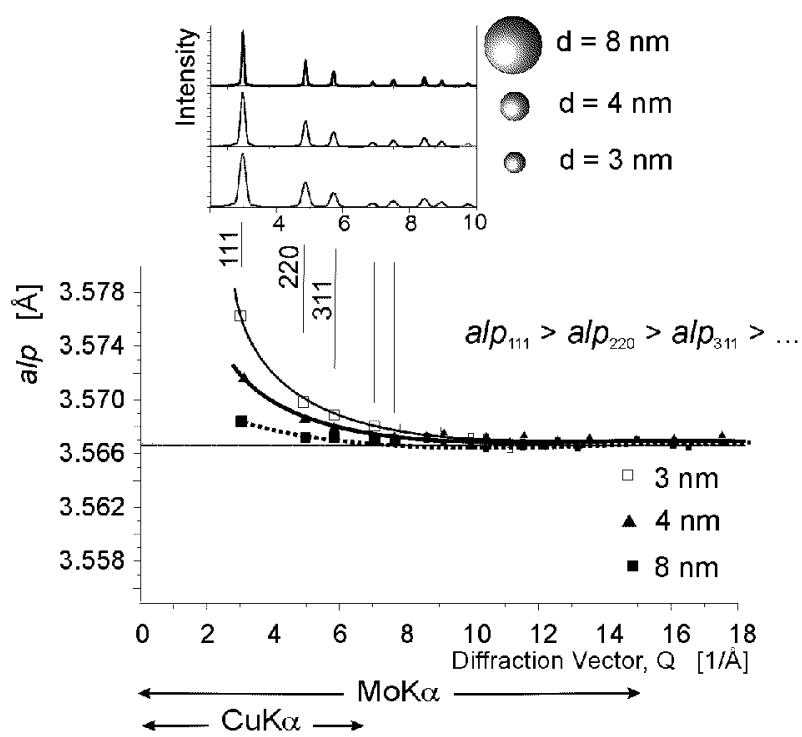

Fig. 7. alp values calculated from individual Bragg reflections of the theoretical diffraction patterns of spherical diamond nanocrystals of 3,4 , and $8 \mathrm{~nm}$ in diameter.

equal the real $a$ value at large diffraction vectors (Fig. 7). Therefore, to derive the $a$ value of the crystal lattice of very small crystallites, diffraction patterns obtained at very large $Q$ 's are necessary.

From the above (Figs. 6 and 7 ) it follows that for a nanocrystal with a perfect, uniform structure its lattice parameter can be derived from powder diffraction patterns using two methods:

(1) a refinement of the real lattice parameters using only the part of the pattern with large $\boldsymbol{Q}$-values (above about $10 \AA^{-1}$ for grains of several nm in size) or

(2) a determination of the alp values for different reflections and matching them with the results of the theoretical predictions obtained for appropriate models. (This method requires the knowledge about the grain size distribution function, which is usually known only approximately.)

A series of our powder diffraction experiments performed in a large $\boldsymbol{Q}$ range for nanocrystalline $\mathrm{SiC}$, diamond, and $\mathrm{GaN}$ particles showed that the type of the alp $\boldsymbol{Q} \boldsymbol{Q}$ relations derived for models of nanocrystals with a perfect crystal lattice is similar to the experimental ones. However, the measured shifts of the Bragg reflections from their proper (i.e. expected for a unique and crystallographically uniform structure) positions are much larger (particularly for small $\boldsymbol{Q}$ 's) than those calculated for nanocrystals with a perfect (unstrained) lattice: for the (111) reflection and $8 \mathrm{~nm}$ diameter $\mathrm{SiC}$ grain the discrepancy is $0.23 \%$ as compared to $0.08 \%$ for a perfect $\mathrm{SiC}$ nanocrystal (cf. Sec. 6.1). This implies that the crystallographic 
structure of nanocrystals is not a uniform perfect lattice such as the one in the bulk material; a nanocrystal is more than just a small single crystal. Accordingly, more complex models of the particles need to be used to evaluate the structure of real nanograins. Therefore, in our further study, we applied the concept of the alp parameters for models with a strain in the surface shell of the grains.

\section{Surface strain in nanocrystals: the concept of the surface free energy}

Non-uniformity of the atomic structure of nanocrystals is obviously caused by the surface of the grains being either reconstructed or under stress. In this work we analyze the atomic structure of nanocrystals based on (with reference to) the general concept of the presence of a surface tension in small liquid droplets and surface stresses in solid particles. A strain present at the surface of small particles is expected to create an internal pressure inside the grain. In this work we assume that the strains are not confined to the outmost layer but propagate to some distance towards the center of the particle.

The theory of surface tension has been developed for liquids but, with some modifications, can also be applied to solids as well $[6,15,22-24,26,27,29]$. The actual atomic structure of a crystallite (of its interior and surface) is a compromise between:

(i) a tendency to reduce the total surface area of the particle (a tendency to adopt the spherical shape [35]) and

(ii) a tendency to reduce the total number of broken bonds at the surface (a tendency to flatten the surface demonstrated by an enlargement of the crystal facets with the lowest free energy at the expense of those with higher ones).

A quantitative description of the surface free energy in solids is very complex. Evaluation of the influence of the surface effects on the atomic structure of a particle can be done using a LaPlace-type law keeping in mind, however, that a derivation of the surface tension coefficient $\gamma$ from the lattice contraction is not fully applicable to solids.

A presence of an internal pressure in the interior of a particle should be detectable through the corresponding change $\Delta a$ of the lattice parameter $a$. In a small crystal of radius $r$ this change can be expressed as a function of the surface stress $f: \Delta a / a=(3 / 2) r f_{r r} / B_{0}$, with $f_{r r}$ - the "mean" surface stress in the radial direction, $r$ - the particle radius, and $B_{0}$ - the bulk modulus. An appropriate experiment designed to evaluate the surface stress would then measure the contraction of the crystal lattice as a function of the particle size. No systematic studies of that type have, however, been reported yet.

The theory of surface tension has been developed for continuous media. The theory does not concern any specific atomic structure of the surface and, also, it ignores the non-tangential components of the surface stresses present in real 
materials. In nanomaterials, where the size of the grains is not much larger than the single interatomic distances, the surface cannot be considered as being a smooth layer which surrounds a certain liquid or solid volume and has a zero thickness. In a nanograin the surface stresses are located at the particle face and its vicinity, so one should talk about a surface shell rather than a two-dimensional surface assumed in the theory of the surface tension.

\section{Effect of a presence of surface strain on alp}

According to the LaPlace-type law the lattice of a nanograin core can either be expanded or compressed hydrostatically (relative to that in large crystals) due to the presence of the surface free energy [22-29]. Based on the above we assumed that the structure of the grain core is uniform and can be described unambiguously by the space group and the lattice parameters. The surface structure is not clearly defined and we can apply only conceptual models of the nanoparticle surface layer, for example:

(i) The structure of the surface shell of a nanograin resembles that of the grain core: it has well defined thickness, symmetry, and interatomic distances;

(ii) A radial strain field exists in the vicinity of the surface: the interatomic distances decrease or increase from the surface towards the particle center;

(iii) The atomic structure of the surface is randomly disordered (forming a glass-like phase).

In models (i) and (ii) a presence of long-range ordering of the surface atoms is assumed, while in model (iii) only a short-range order in the surface layer is present. In any case, the surface is a "low-dimensional structure" and has a defined thickness, but it also contains strains and, thus, cannot be unambiguously defined and represented by unit cell parameters. No experimental methods, which would allow for a direct analysis of such complex structures of nanocrystals, are available at this moment [36-38]. The analysis of the conditions required for ordering of atoms in three-dimensional crystal lattices, and application of the general theory of the origin of the surface strain from the internal pressure in the grain interior could be helpful for predictions of the surface structure of nanocrystalline grains. No general theory or model of the surface of nanocrystals is available yet. In this work we applied the model of a nanocrystal where the grain core has a uniform crystallographic structure and is unambiguously characterized by the lattice parameter $a_{0}$. We assumed that the atomic structure of the surface is strongly correlated with the parent structure of the grain: it is basically the structure of the core but centro-symmetrically deformed, compressed (Fig. 8) or expanded due to a presence of the surface strain. According to the model, the term "lattice parameters" may neither be applied to the whole nanocrystal nor to the surface layer. No common, specific interatomic distances can be assigned to the surface shell. However, to 
simplify the description of the model, we introduce the parameter $a_{\mathrm{S}}$ which corresponds to the interatomic distances at the outmost atomic layer of the particle (Fig. 8). Assuming that the arrangement of the atoms in the surface shell is similar to that in the grain core, the value of $a_{\mathrm{s}}$ can be expressed as a fraction (in \%) of the lattice parameter $a_{0}$. The actual values of the interatomic distances within the surface shell can be expressed as a function of the distance from the particle center and vary between $a_{0}$ in the grain core (at distances $r<R_{0}$ ) and $a_{\mathrm{s}}=a_{0} \pm \Delta a$ (for $r=R$ ). The sign in front of $\Delta a$ depends on whether the surface is compressed $(-)$ or expanded $(+)$ relative to the grain core. The ratio $\Delta a / a_{0}$ is quantifying the surface strain.

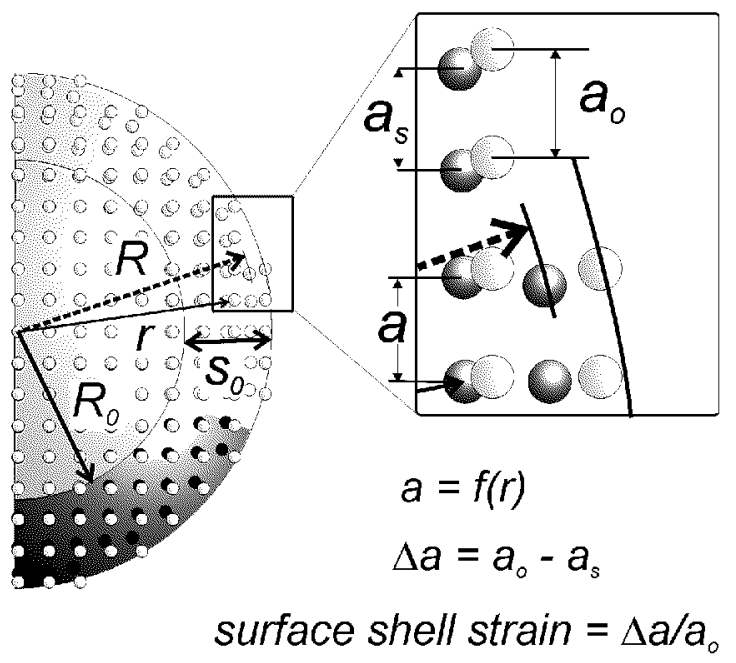

Fig. 8. Tentative model of a nanocrystal with strained (compressed) surface layer. $R_{0}$ - radius of the core, $R$ - radius of the grain, so - thickness of the surface layer, $r$ - distance from the center, $a_{0}$ - interatomic distance in the relaxed lattice, $a_{\mathrm{s}}$ interatomic distance at the surface.

Our model of a cubic lattice nanoparticle with and without the tensile strain in the surface shell is shown in Figs. 9a and b, respectively. A presence of strain in the grain surface layer, the surface shell, leads to a deformation of the atomic planes (Fig. 9a). As shown in the figure, the atomic planes lose their planarity and mutual parallelity. As a consequence, the interplanar spacings in such nanocrystals lose their unambiguous meaning. Also, the conventional description of a crystal lattice based on the definition of a unit cell cannot be applied to the whole crystallite although it still can be used for the description of the grain core structure. A diffraction on such crystal-like objects may not be based, however, on the Bragg approximation: the concept of interplanar spacings is meaningless for non-planar (bent) surfaces. However, for the sake of convenience and consistency with the 
description of the diffraction effects on nanocrystals having a perfect crystal lattice (cf. Secs. 2.2.2 and 3) we use the classical description of the diffraction patterns also for our alp concept. The diffraction patterns calculated for the models of an $8 \mathrm{~nm}$ diameter $\mathrm{SiC}$ nanocrystals with a tensile, none, and compressive uniform strain in the surface shell ( $\Delta a / a_{0}=$ const) are shown in Figs. 10a, b, and c, respectively. The strain in the surface shell shifts the positions and changes the symmetry of the Bragg reflections. Since asymmetric peaks do not have uniquely defined positions, those were determined based on the location of both the maximum and the weighted center of the individual reflections. The character of the dependence of the calculated alp values on $\boldsymbol{Q}$ is similar for both methods, although specific values of $\boldsymbol{Q}$ are somewhat different.

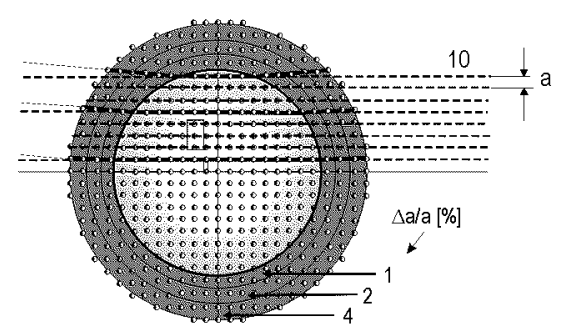

(a)

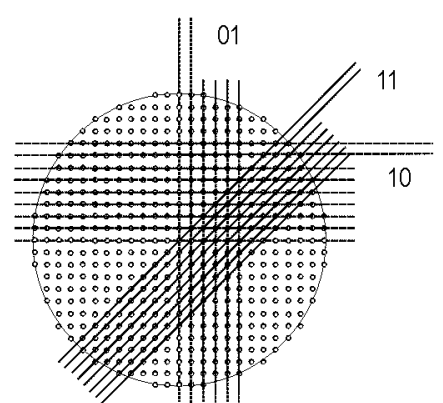

(b)

Fig. 9. Schematic representation of cubic nanostructures: (a) grain with strained (expanded) surface shell, (b) grain with uniform, perfect lattice.

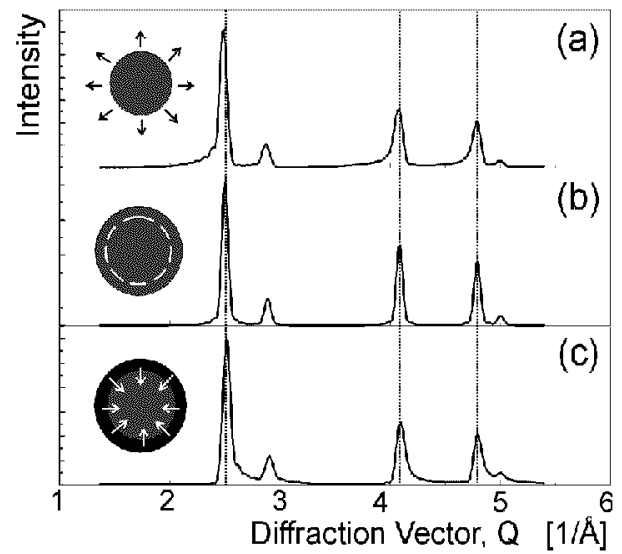

Fig. 10. Diffraction patterns of a $8 \mathrm{~nm} \mathrm{SiC} \mathrm{grain} \mathrm{with} \mathrm{the} \mathrm{surface} \mathrm{shell} \mathrm{of} 0.7 \mathrm{~nm}$ in thickness, calculated for different magnitudes of strain in the surface layer. (a) $\Delta a / a=$ $+10 \%$, (b) $\Delta a / a=0 \%$, (c) $\Delta a / a=-10 \%$. 


\section{6. alp analysis of powder diffraction data}

To determine the alp- $\boldsymbol{Q}$ relation for our theoretical and experimental patterns, the diffraction peaks were fitted using a standard shape function (pseudo-Voigt) a vailable in every Rietveld program. The examples of Bragg-like reflections (111 and 246) calculated for 6 and $12 \mathrm{~nm}$ diameter SiC nanocrystals are shown in Figs. 11a and b, respectively. The patterns were calculated both for models with a relaxed, perfect lattice and for nanocrystals with a strained surface shell. Both the shapes and positions of the reflections change with the grain size, a presence of strain, and the diffraction vector $\boldsymbol{Q}$ (Fig. 11).

$\mathrm{SiC}$

(a)

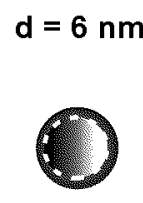

(b)
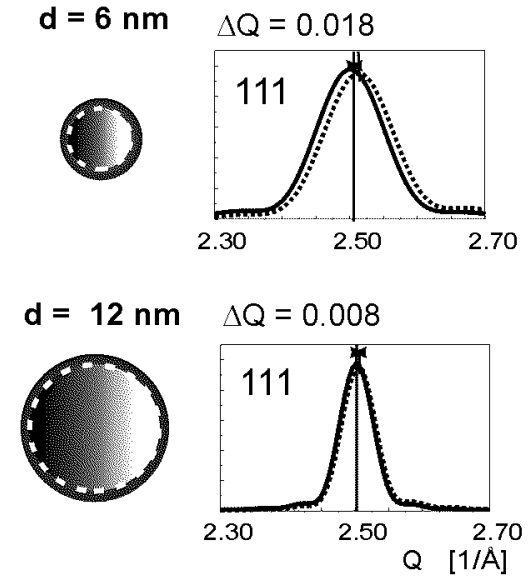
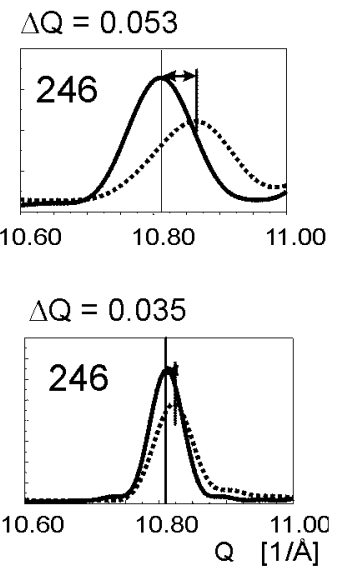

Fig. 11. Bragg-like intensity curves calculated for models of SiC nanocrystals with a relaxed lattice (solid lines) and compressed surface shell (dotted lines) for two different reflections, (111) and (246). (a) Grain diameter $6 \mathrm{~nm}$, (b) grain diameter $12 \mathrm{~nm}$. The thickness of the surface shell, $s_{0}=0.7 \mathrm{~nm}$, the strain $\Delta a / a_{0}=5 \%$.

When designing a diffraction experiment one has to evaluate the resolution required for the detection of the expected diffraction effects. In our case (the analysis of the alp- $\boldsymbol{Q}$ relations) we have to focus either on recording in a wide $\boldsymbol{Q}$-range or on a precise measurement of the positions of relatively low $\boldsymbol{Q}$-value reflections. We performed a number of different experiments using synchrotron sources and making different compromises: to measure at a very large $\boldsymbol{Q}$-range (up to $25-30 \AA^{-1}$ ) with a moderate resolution we used the Station ID11 at ESRF and Station BW5 at HASYLAB (the wavelength of $0.1-0.2 \AA$ ); to get a very good resolution we used the wavelength of $0.5-0.7 \AA$ at SNBL Station at $\operatorname{ESRF}\left(Q_{\max }=\right.$ $\left.10-15 \AA^{-1}\right)$. In addition to the advantage of the short wavelength radiation not available with sealed $\mathrm{X}$-ray tubes, the powder diffraction instruments installed at synchrotron sources have usually much better angular resolution than those available in conventional laboratories. 


\subsection{Effect of the model parameters on alp- $Q$ relation}

We performed a systematic theoretical study of diffraction by nanocrystalline materials using models with different thickness of the surface layer, various magnitudes of the surface strain, and diverse gradients of the strain in the shell. The dependence of the alp on $\boldsymbol{Q}$ for $\mathrm{SiC}$ nanocrystals with the same strain of $+5 \%$ and the surface shell thickness of $0.7 \mathrm{~nm}$ but different grain size is shown in Fig. 12. For a reference, the graphs contain also the $a l p-\boldsymbol{Q}$ results for the corresponding models with a fully relaxed (unstrained) lattice (cf. Fig. 7). The calculated deviations of alp from the core value of $a_{0}$ for strained grains are much larger than for those with the relaxed lattice. The difference between the alp and $a_{0}$ values increases with a decrease in the grain size, reaching as much as $1.7 \%$ of the lattice parameter of the relaxed $\mathrm{SiC}$ crystal for the $3 \mathrm{~nm}$ particle. These results are not surprising: for a $0.7 \mathrm{~nm}$ thick shell the strained fraction of the grain contains from 85 percent (for the smallest, $3 \mathrm{~nm}$ in diameter) to 30 percent (for the largest, $12 \mathrm{~nm}$ ) of all the atoms in the particle. The results shown in Fig. 12 illustrate the consequences

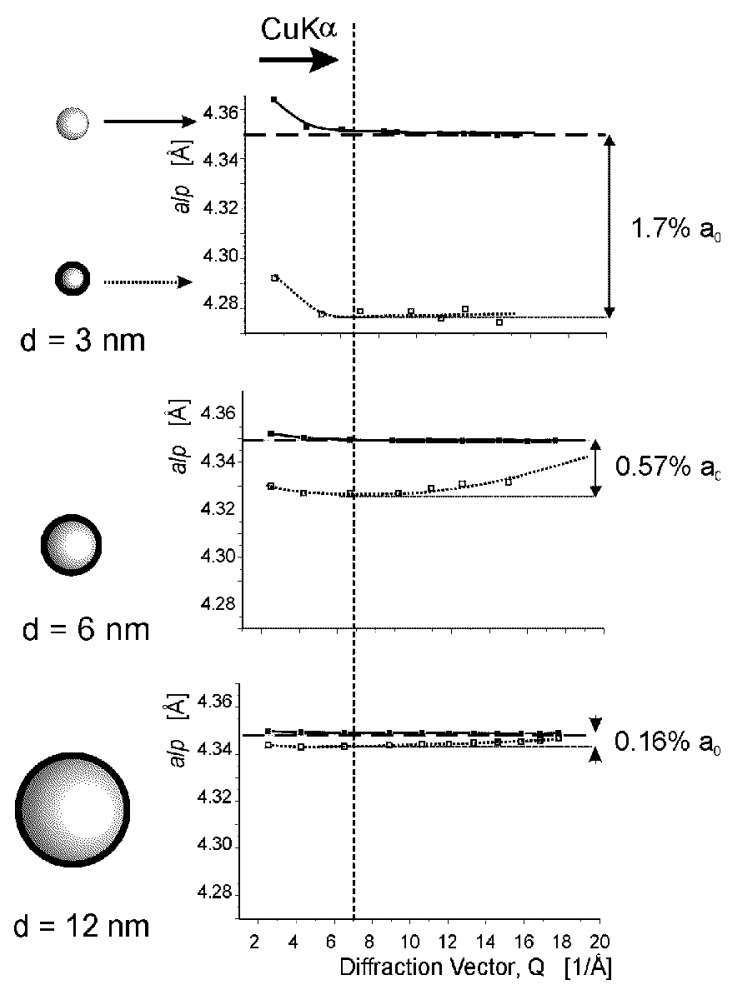

Fig. 12. alp values calculated theoretically for nanocrystalline cubic $\mathrm{SiC}$ of different grain size. $a_{0}=4.349 \AA, \Delta a / a_{0}=-5 \%, s_{0}=0.7 \mathrm{~nm}$. 
of using a standard powder diffraction technique combined with commonly applied radiation sources. The widely used $\mathrm{Cu} K_{\alpha}$ X-ray radiation source allows for a collection of diffraction data in the $Q$-range up to only $5-7 \AA^{-1}$. For strained particles shown in Fig. 12 a routine elaboration of such data using the Rietveld program would yield one value of the lattice parameters differing from $a_{0}$ of the relaxed lattice in the grain core from 0.16 up to about $1.7 \%$ (Fig. 12).

A single alp value determined from a diffraction pattern has no specific meaning: only a set of alp values in a wider $\boldsymbol{Q}$ range can provide a meaningful information about the structure of the nanocrystal. The alp values at sufficiently high $\boldsymbol{Q}$-values correspond to the lattice parameters of the core of the particle, i.e. the relaxed lattice. The experimentally determined dependence of alp on $\boldsymbol{Q}$ combined with numerical simulations can provide a meaningful information about the structure of the surface shell of the nanocrystal. Examples of alp- $\boldsymbol{Q}$ data determined from theoretical diffraction patterns calculated for different models of $\mathrm{SiC}$ nanocrystals are shown in Figs. 13-15. The sensitivity of the alp values on the parameters of the model is quite high: differences as small as $1 \%$ in the lattice strain and $0.1 \mathrm{~nm}$ in the thickness $s_{0}$ of the surface shell are clearly visible (Figs. 13-15). Although in all models the lattice parameter of the grain core is the same $\left(a_{0}=\right.$ $4.349 \AA$ - the relaxed lattice) the alp values practically never reach $a_{0}$ except for greater $Q$ 's and larger surface strains. This shows that conventional diffraction experiments (low intensity laboratory sources, $\mathrm{Cu} K_{\alpha}$ or Mo $K_{\alpha}$, maximum $\boldsymbol{Q}$

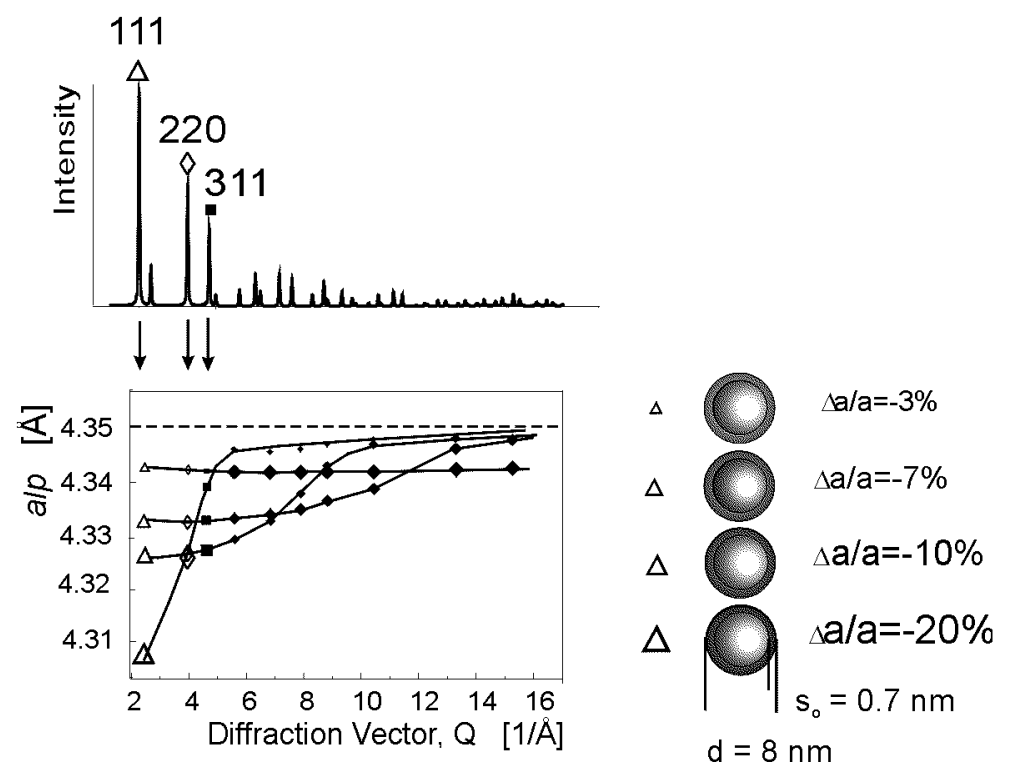

Fig. 13. alp- $Q$ plots calculated theoretically for spherical $8 \mathrm{~nm}$ diameter SiC nanocrystals for different values of the compressive stress in the surface shell. $s_{0}=0.7 \mathrm{~nm}$. 

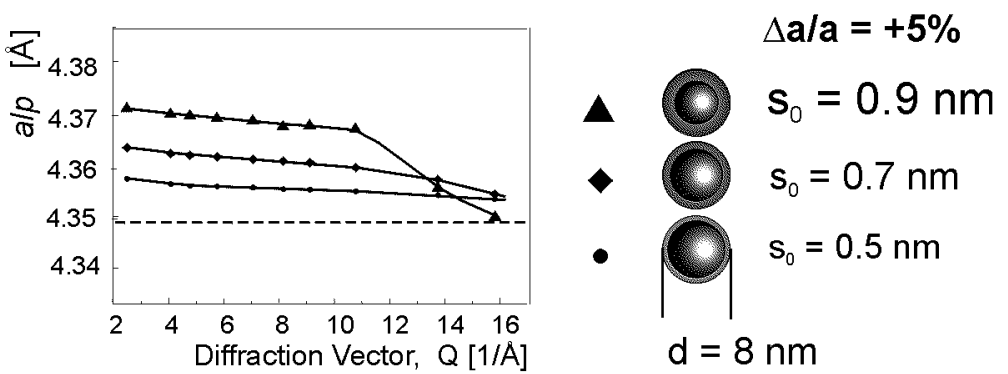

Fig. 14. alp- $\boldsymbol{Q}$ plots calculated theoretically for spherical $8 \mathrm{~nm}$ diameter SiC nanocrystals for different thickness of the surface shell. $\Delta a / a_{0}=+5 \%$.
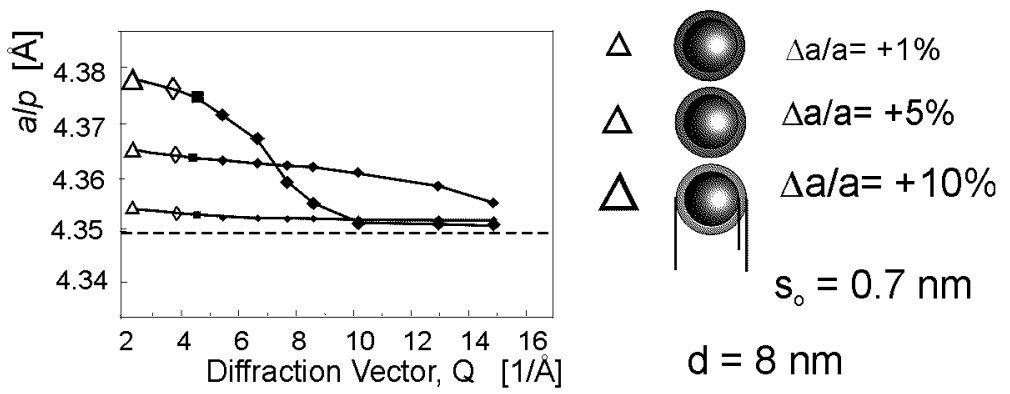

Fig. 15. alp- $\boldsymbol{Q}$ plots calculated theoretically for spherical $8 \mathrm{~nm}$ diameter $\mathrm{SiC}$ nanocrystals for different strain values in the surface shell.

range of 7 and $15 \AA^{-1}$, respectively, elucidation of the common lattice parameters from the entire diffraction diagram) do not allow for a unique interpretation of the lattice parameters calculated from the Bragg reflections.

In our theoretical computations both the strain in the surface shell and the lattice parameters of the grain core are the variable parameters of the core-shell model. For that reason the interpretation of the alp- $\boldsymbol{Q}$ results needs to be done with caution. The plots presented in this paper have well defined characteristic shapes which are sensitive to the lattice parameters of the grain core and the strain in the surface shell. The atomic structure of the nanograins does not form planes but bent surfaces (Fig. 9), so using the Bragg relations in the calculations of the alp values yields some average spacings between the deformed planes and not the real interplanar distances. Nevertheless, powder diffraction data combined with our alp methodology (based on geometrical factors alone) can provide quite realistic but still approximate model of the core-shell structure of the nanocrystal. Based on our analysis we conclude that the correct values of the lattice parameters of the grain cores can be determined from the diffraction experiments performed in a wide range of the $\boldsymbol{Q}$ vector. To determine the specific positions of atoms in the surface shell an analysis of the relative intensities of the Bragg-like reflections is 
necessary. Currently we are investigating an alternate approach to determination of interatomic distances in these materials using the neutron diffraction and the so-called "real-space Rietveld refinement" technique [39, 40].

\subsection{Application of the concept of alp to the evaluation of experimental diffraction data of nanopowders}

We applied our methodology for evaluation of the experimental diffraction data of different nanocrystals. The powder diffraction patterns in the range up to $Q=20 \AA^{-1}$ were obtained using a short wavelength synchrotron radiation sources (at BW5 Station at HASYLAB, and ID 11 and SNBL powder diffractometers at ESRF). At the present stage of our studies we claim a qualitative agreement between the alp- $\boldsymbol{Q}$ plots determined from the experimental powder diffraction data of nanocrystalline $\mathrm{SiC}, \mathrm{GaN}$ and diamond, and our theoretical calculations. For example, we deduced that a tensile strain exists in the surface shell of nanocrystalline $\mathrm{SiC}$ (compare Figs. 15 and 16). Similar strain is present in diamond nanoparticles. Opposite in sign, compressive strain at the surface is present in a much softer material, nanocrystalline GaN (Fig. 17).

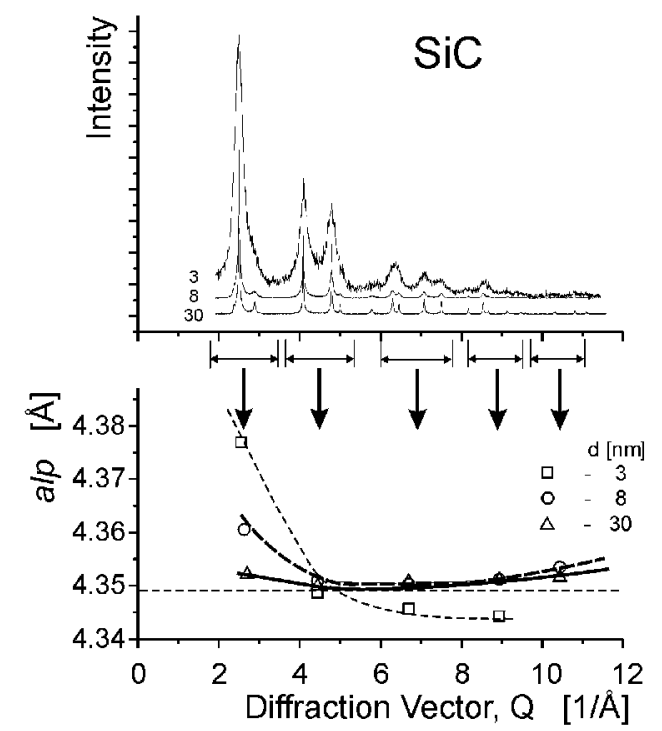

Fig. 16. Experimental diffraction patterns of nanocrystalline $\mathrm{SiC}$ powders with a verage grains of 3, 8, and $30 \mathrm{~nm}$ in diameter (upper graph) and their corresponding alp plots (lower graph). The measurements were performed with the powder diffractometer at SNBL Station, ESRF, Grenoble, with $\lambda=0.7 \AA$ radiation source.

The surface of a nanoparticle is in a metastable state and, thus, is strongly dependent on its surrounding. The surface exposed to a gas, liquid, or solid medium can adsorb foreign atoms or even undergo a chemical reaction. Interaction of the 


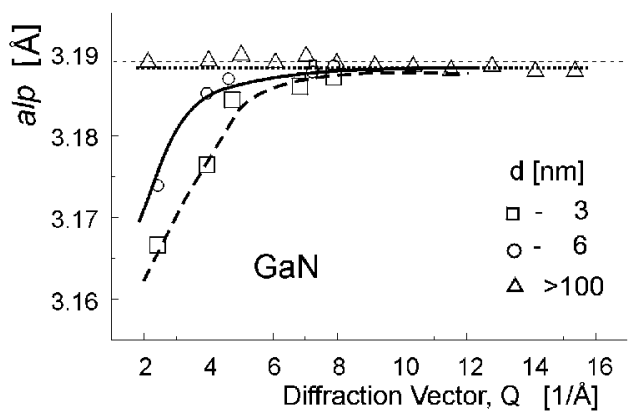

Fig. 17. alp- $\boldsymbol{Q}$ plots obtained experimentally for GaN nanocrystals. The measurements were performed with the powder diffractometer BW5, HASYLAB at DESY, with $\lambda=$ $0.12 \AA$ radiation source.

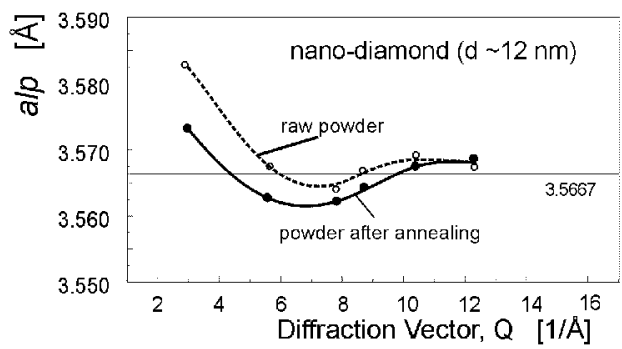

Fig. 18. alp- $\boldsymbol{Q}$ plots obtained experimentally for diamond nanocrystals aged in the air (dotted line) and annealed in vacuum at $400^{\circ} \mathrm{C}$ for $4 \mathrm{~h}$ (solid line). The measurements were performed at powder diffractometer BW5, HASYLAB at DESY, with $\lambda=0.12 \AA$ radiation source.

surface with foreign atoms must affect the atomic structure of the surface. We have examined this problem in a series of experiments performed for nanocrystalline powders of diamond and $\mathrm{SiC}$. The powders were annealed at elevated temperature under vacuum or used as components of ceramic composites. An example of the alp values vs. the $\boldsymbol{Q}$ vector for raw and annealed diamond powders is given in Fig. 18 .

In this work we did not attempt to accomplish a quantitative evaluation of the structure of nanocrystals based on the observed changes of alp values with $\boldsymbol{Q}$; additional measurements of specific physical properties of our materials are necessary to determine what kind of changes occurs at the surface and how much they affect the structure of the grain core. The alp concept provides a sensitive tool for investigation of the structural features of very small, nanosized crystallites. From our preliminary calculations based on the models of nanocrystals with different surface strain and thickness we conclude that annealing of diamond powders leads to a significant reduction in the strain present in the surface shell without much 
change of the structure of the grain core. This is indicated by the strongest changes of the alp occurring in the low $\boldsymbol{Q}$ range (which is very sensitive to the structure of the surface shell) and a convergence of the alp's of raw and processed powders at larger $\boldsymbol{Q}$ values (Fig. 18).

The results of our experimental work presented in this paper are only preliminary. A more definite description of the structure of nanosize particles requires both additional examination of the physical properties and structure of the materials (using techniques sensitive to the local atomic configuration, like Raman or IR spectroscopy) and more extensive modeling efforts that would include different strain fields both in terms of strength and distribution in the surface and core of the grain.

\section{Summary and conclusions}

In this work we showed that the conventional tools developed for elaboration of powder diffraction data are not directly applicable to nanocrystals:

(i) Nanoparticles have a complex structure that resembles rather a two-phase than a uniform, one-phase material. Therefore the definitions and parameters used for characterization of the atomic structure of simple, crystallographically uniform phases are insufficient for the description of the complex structure of nanocrystals (Note: the core-shell model of a nanocrystal applied in this work is a kind of a two-phase system that exhibits unique diffraction features. One has to remember, however, that although the two-phase model of nanocrystals is practical, it defies, in fact, the basic definitions of a crystallographic phase.);

(ii) Conventional powder diffraction techniques and appropriate methods of their elaboration have been developed for materials showing a perfect, three-dimensional, periodic order in an infinite crystal lattice. A nanocrystal is a finite in size crystal where the effect of boundaries cannot be ignored. For very small, several $\mathrm{nm}$ in diameter crystallites the number of atoms at the surface can exceed the number of atoms in the grain core. The structural and other properties of the surface atoms can dominate over the effects imposed by the bulk atoms. This effect is clearly observed in our diffraction experiments.

This work is dedicated to the methodology of elaboration of powder diffraction data of nanocrystals, not to the determination of the atomic structure of some specific materials. We have shown the limitations of a conventional structural analysis approach and proposed a new method of evaluation of diffraction data of nanosize polycrystalline materials. We have demonstrated a qualitative agreement between the experimental results and those obtained by numerical modeling using our alp concept methodology. A more complete evaluation of powder diffraction data of specific nanocrystalline materials requires a wider spectrum of structural models as well as accounting for the shape and size distribution of nanocrystals in real materials. The work on these issues is in progress. 
In this paper we have shown the limits of applicability of well-established methods of structural analysis of materials by the diffraction techniques. When it comes to nanomaterials there are obviously limitations of other commonly applied techniques and procedures used in determination of different physical properties of materials. A development of new experimental and theoretical tools for investigation of unique properties of nanocrystals is a challenge and a must.

\section{Acknowledgments}

This work was supported by the State Committee for Scientific Research grant PBZ/KBN-013/T08/30, the Polish-German project POL-00/009 and in part by the EC grant "Support for Centers of Excellence" No. ICA1-CT-2000-70005, DESY-HASYLAB Project II-99-053, ESRF project HS-1463. Support from the Office of Physical and Biological Research of NASA is gratefully acknowledged.

\section{References}

[1] H. Fujita, Mater. Trans. JIM (Trans. Japan Inst. Met.) 38, 659 (1997).

[2] H. Fujita, J. Electron Microsc. 48, Suppl, 983 (1999).

[3] H. Fujita, Mater. Trans. JIM (Trans. Japan Inst. Met.) 35, 563 (1994).

[4] Nanomaterials: Synthesis, Properties and Applications, Eds. A.S. Edelstein, R.C. Cammarata, Institute of Physics Publishing, Bristol 1996.

[5] Physics of New Materials, Ed. F.E. Fujita, Springer Series in Materials Science, Springer-Verlag, Berlin 1998.

[6] Interfaces in Materials, Ed. M.J. Howe, Wiley, New York 1997.

[7] Non-equilibrium Processing of Materials, Ed. C. Suryanarayana, Pergamon, Amsterdam 1999.

[8] Z.L. Wang, Characterization of Nanophase Materials, Wiley-VCH, Weinheim 2000

[9] R.S. Averback, Z. Phys. D 26, 84 (1993).

[10] Ph. Buffat, J.-P. Borel, Phys. Rev. A 13, 2287 (1976).

[11] Amorphous and Nanocrystalline Materials: Preparation, Properties and Applications, Eds. A. Inoue, K. Hishimoto, Springer-Verlag, Berlin 2001.

[12] S.B. Qadri, J. Yang, B.R. Ratna, E.F. Skelton, J.Z. Hu, Appl. Phys. Lett. 69, 2205 (1996).

[13] M.R. Silvestri, J. Schroeder, J. Phys., Condens. Matter 7, 8519 (1995).

[14] S.H. Tolbert, A.P. Alivisatos, J. Phys. D 26, 56 (1993).

[15] D. Wolf, K.L. Merkle, in: Materials Interfaces: Atomic Level Structure and Properties, Eds. D. Wolf, S. Yip, Chapman and Hall, London 1992, p. 87.

[16] J. Harada, K. Ohshima, Surf. Sci. 106, 51 (1981).

[17] C. Solliard, M. Flueli, Surf. Sci. 156, 487 (1985).

[18] J. Woltersdorf, A.S. Nepijko, E. Pippel, Surf. Sci. 106, 64 (1981). 
[19] P.A. Montano, G.K. Shenoy, E.E. Alp, W. Schulze, J. Urban, Phys. Rev. Lett. 56, 2076 (1986).

[20] S.H. Tolbert, A.P. Alivisatos, J. Chem. Phys. 102, 4642 (1995).

[21] F.W.C. Boswell, Proc. Phys. Soc. Lond. A 64, 465 (1951).

[22] J.-P. Borel, A. Chatelain, Surf. Sci. 156, 572 (1985).

[23] R.C. Cammarata, Surf. Sci. Lett. 273, L399 (1992).

[24] R.C. Cammarata, Mater. Sci. Eng. A 237, 180 (1997).

[25] J.J. Gilman, J. Appl. Phys. 31, 2208 (1960).

[26] C.W. Mays, J.S. Vermaak, D. Kuhlmann-Wilsdorf, Surf. Sci. 12, 134 (1968).

[27] R. Shuttleworth, Proc. Phys. Soc. Lond. A 63, 444 (1950).

[28] A.M. Stoneham, J. Phys. C, Solid State Phys. 10, 1175 (1977).

[29] J.S. Vermaak, C.W. Mays, D. Kuhlmann-Wilsdorf, Surf. Sci. 12, 128 (1968).

[30] D.L. Bish, J.E. Post, Modern Powder Diffraction, Reviews in Mineralogy, Vol. 20, Mineralogical Society of America, Washington, DC 1989.

[31] The Rietveld Method, Ed. R.A. Young, International Union of Crystallography, Oxford University Press, Oxford 1993.

[32] H.P. Klug, L.E. Alexander, X-Ray Diffraction Procedures for Polycrystaline Materials, 2nd ed., Wiley, New York 1954.

[33] B. Bondars, S. Gierlotka, B. Palosz, Mater. Sci. Forum 133, 301 (1993).

[34] R. Pielaszek, S. Gierlotka, S. Stel'makh, E. Grzanka, B. Palosz, Defect Diff. Forum 208-209, 487 (2002).

[35] N. Combe, P. Jensen, A. Pimpinelli, Phys. Rev. Lett. 85, 110 (2000).

[36] M.R. Fitzsimmons, J.A. Eastman, M. Mueller-Stach, G. Wallner, Phys. Rev. B 44, 2452 (1991).

[37] J. Loeffler, J. Weissmueller, Phys. Rev. B 52, 7076 (1995).

[38] X. Zhu, R. Birringer, U. Herr, H. Gleiter, Phys. Rev. B 35, 9085 (1987).

[39] S.J.L. Billinge, M.F. Thorpe, Local Structure from Diffraction, Plenum Press, New York 1998.

[40] Th. Proffen, S.J.L. Billinge, J. Appl. Crystallogr. 32, 579 (1999). 